\title{
THE RELATIONSHIP BETWEEN THE CHOICE OF SKETCHING TOOL AND STUDENT DESIGNER'S FLOW
}

\author{
Gavin BARR, Ross MacLACHLAN \\ University of Strathclyde, UK
}

\begin{abstract}
The role of sketching is evolving within contemporary design practice and digital sketching tools are increasingly used by design professionals and students. The promise of efficient rework, presentation aids and a wide range of virtual tools are immediately compelling, but there has yet to be a deeper analysis of their true value with respect to design task engagement and productivity. The study outlined in this paper assesses student product designers' 'design flow' during sketch-based ideation and proposes a relationship for how this influences productivity. The results indicate that digital sketching tools provide a more immersive environment than the conventional analogue sketch, but that this has a negative impact on productivity. The study provides insights into how this may impact design educators going forward and how digital sketching tools could be improved for initial ideation activities.
\end{abstract}

Keywords: Design flow, design research, sketching, ideation, evaluation, design tool

\section{INTRODUCTION}

The role of sketching in design practice and education is evolving but remains as essential in developing, capturing and communicating design intent. Recently digital sketching tools are more desirable with vastly improved accuracy and affordability. Beyond replication of the traditional sketching experience, perceived paperless advantages such as efficient rework, presentation aids and a wide range of virtual tools, are further convincing. Many students are independently persisting with digital platforms and hardware for sketching in evolving their design practice. Despite these points, digital sketch tools are rarely prescribed or promoted in higher education. There is no evidence that, beyond the convenience of working entirely in the digital domain, these tools actually improve design task performance. There may also be suspicions that students can become 'tool focused' rather than on the problem or productivity. To provide insights for enhanced support for sketching in design engineering education, this study compares traditional and digital sketching tools used by senior product design students, with regard to task engagement and their creative productivity.

\section{LITERATURE REVIEW}

Previous studies have provided arguments for and against digital sketching tools, taking multiple approaches towards the issue. Jonson [1] found that young designer's used sketching and CAD tools equally during ideation, contradicting the widespread assumption that traditional pen and paper sketching was fundamental to the ideation phase of the design process. Others have also argued for the introduction of new digital tools, with Coyne et al. [2] suggesting they could prompt new practices and representations in design. More recent studies appear to agree with these views, concluding that digital tools improve accuracy in representing form, increase confidence in creating lines and reduce the time taken to complete a sketch [3]. However, studies directly comparing both methods have found that student designers tend to become 'tool focused' when sketching digitally, rather than focusing on driving the design forward [4]. This tool focus is said to disrupt the reflective 'conversation' between the designer and their ideas and ultimately results in less productive ideation sessions. In summary, whilst it is clear that digital sketching is embedding in industrial and student practice, studies on the true cognitive and productivity benefits are not fully understood in the context of design education. 


\subsection{Flow}

As it is desirable to improve the quality and efficiency of the design process, it can be argued that designers should be in an optimal state of mind when designing. This optimal cognitive state is regarded in most fields as 'flow', in lay terms as being "in the zone". Flow is an immersive experience characterised by intense focus on a task, losing track of time and having no fear of failure [5]. It occurs when a person exercises a high level of skill during a challenging activity, allowing them to become completely absorbed in the task at hand. The study of flow was pioneered by Mihaly Csikszentmihalyi, a psychologist who wanted to understand why creatives became completely immersed in their work, forgoing hunger and rest, only to lose interest after the work was finished. He concluded that flow is an 'autotelic state' in which a person is willing do something for its own sake, rather than to achieve an external goal [5]. Increasing the number of flow occurrences experienced is beneficial as this has been linked with positive affect, skill development, longer commitment to an activity and higher achievement within education [6] [7]. In primary education, these findings have been utilised to create environments and programmes designed to increase the potential to experience flow and have helped students to identify their interests and develop their capabilities [8]. Flow has also been considered in other disciplines, such as software design, where it is employed as a measure of user engagement for improving their experience [9]. Therefore, the designer's flow when using digital sketching software is of particular interest.

Within design research flow has been used to assess designer experience in prototype design environments, with Dorta et al. [10] suggesting that high flow indicates a 'rich' ideation session. They conceptualised Design Flow Theory; a repeating cycle of cognitive states experienced by the designer when generating ideas, rather than a single flow experience [10]. This cycle is characterised by a zone of stress when trying to think of a suitable idea, followed by flow when an idea has been selected and is externalised, and concludes with a zone of control and relaxation as the idea is solidified. Within this theory, it is suggested that designer flow is linked to the quality and productivity of ideation [10].

Despite the development of design flow theory, the study of flow in engineering design has been limited by measurement methods which are said to be intrusive and disruptive to the ideation process. However, Safin et al. [11] have recently proposed a new method for non-intrusively assessing flow with increased granularity, offering new opportunities for analysing existing design tools. By employing this method, the study outlined in this paper aimed to compare traditional and digital sketching tools in order to establish how choice of tool influences the designer's flow experience and how this ultimately affects their productivity during ideation.

\section{RESEARCH DESIGN}

An ideation task was individually completed by 20 students, 10 using analogue sketching tools (pen and paper) and the other 10 using digital sketching tools (sketching tablets etc.). The task was to generate concepts for a sports watch aimed at cycling enthusiasts using any type of technology available and aiming to have unique features to stand out in the market. Based on pilot study feedback the experiment length was set at 7 minutes. This gave participants enough time to process the brief and generate multiple concepts. Participants were not told of the 7 minute length and were asked to keep generating ideas until stopped. This enabled measurement of time distortion, a key indicator of flow. Participants were senior students and recent alumni from an undergraduate product design engineering programme, each having at least 2 years general design sketching experience and, in the case of digital sketchers, had used their selected tool for a minimum of 6 months. The experiments were conducted in private meeting rooms which provided a quiet, stable environment with no external distractions. A camera was set-up facing the participant, capturing their facial expressions and movement during the activity.

The study used a multi-stage evaluation to measure characteristics of flow before, during and after the experiment. A pre-task evaluation captured participant interest, perceived control, perceived challenge and perceived skill on a 5 point Likert scale. Additional data on sketching experience and the chosen tool was also recorded. Immediately after the experiment, participants completed a post-task evaluation which combined Keller and Bless's [12] time perception test and Mayer's Flow Scale [13]. This captured data on twelve individual characteristics of flow including time distortion. Using a modified version of the Design Flow 2 method, participants re-watched the design activity footage and recorded their perceived challenge and skill on an 11 point Likert Scale at 15 second internals. The participants cognitive experience was tracked as it fluctuated throughout the design task whilst not intruding during the actual activity; a particular strength of the Design Flow 2 method [11]. Finally, 10 minutes after the 
activity ended, participants completed an end of task reflection form which recorded challenge, skill, absorption, timelessness and effort to focus using a 5 point Likert scale to create a comparison to the original pre-task evaluation. Following the completion of the experiment, the researcher recorded each participant's fluency, the total number of different relevant ideas and elaboration, the number of different ideas used in working out the details of an idea. The extensive evaluation method allowed for a significant data collection process which aided accurate identification of flow during the activity without interruption.
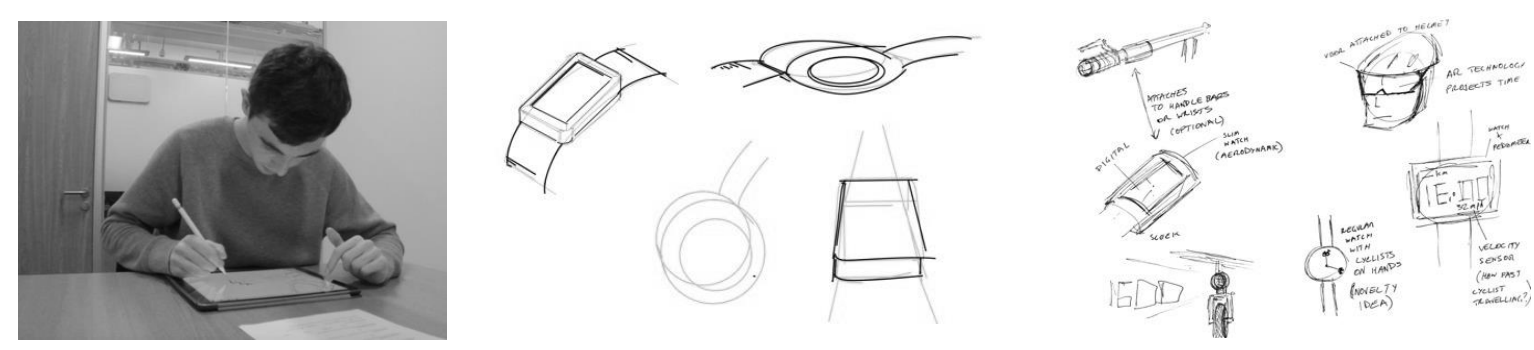

Figure 1. Example of video, digital and analogue sketch output

\subsection{Pre-Task Evaluation Results}

Table 1. Pre-task evaluation results

\begin{tabular}{|l|c|c|c|c|c|}
\hline & Analog & SD & Digital & SD & $p$ Value \\
\hline Interest & 3.9 & 0.737 & 3.8 & 0.788 & 0.773 \\
\hline Perceived Control & 3.1 & 0.875 & 3.7 & 0.674 & 0.104 \\
\hline Perceived Challenge & 3.3 & 1.059 & 3 & 0.816 & 0.488 \\
\hline Perceived Skill & 3.2 & 0.918 & 3.4 & 1.173 & 0.676 \\
\hline
\end{tabular}

Pre-task evaluation indicates interest in the task; the flow state requires a person to be interested in the task [5]. For this study, the digital sketching tool was not standardised. The most popular hardware was the 12.7" iPad Pro and Apple Pencil $(n=4)$ along with a Wacom graphics tablet set up $(n=4)$. The most popular software was Autodesk Sketchbook $(n=7)$.

\subsection{Post-Task Evaluation Results}

Mayer's [13] Flow Scale measures 12 characteristics of flow. The outlier regarded the rating for direct feedback which was low in both groups. Intrinsic motivation was significantly higher for digital sketchers. In addition, distraction was found to be lower in the digital group. A time distortion test [12] records an analogue reading on a blank $10 \mathrm{~cm}$ line, which found the digital group experienced a greater sense of timelessness. This corroborated with Mayer's [13] Likert based Flow Scale, though this was not found to be significant.

Table 2. Selected post-task evaluation results

\begin{tabular}{|l|l|l|l|l|l|}
\hline & Analog & SD & Digital & SD & $p$ Value \\
\hline 1. I got involved. & 8.15 & 1.25 & 7.6 & 1.71 & 0.423 \\
\hline 3. I knew clearly what I was supposed to do. & 6.85 & 3.20 & 8.5 & 1.18 & 0.153 \\
\hline 4. I got direct clues as to how well I was doing. & 1.6 & 2.28 & 1.3 & 0.95 & 0.707 \\
\hline 5. I felt I could handle the demands of the situation. & 6.3 & 3.05 & 7.8 & 1.23 & 0.174 \\
\hline 8. I had to make an effort to keep my mind on what was happening. & 3.8 & 2.19 & 2.25 & 1.93 & 0.110 \\
\hline 9. I would do it even if I didn't have too. & 4 & 2.97 & 7.5 & 2.12 & 0.007 \\
\hline 10. I got distracted. & 2.6 & 1.45 & 1.25 & 1.03 & 0.029 \\
\hline 11. Time past slow...fast. & 7.05 & 1.92 & 8.5 & 1.72 & 0.091 \\
\hline
\end{tabular}




\subsection{On-Task Evaluation Results}

Table 3. On-task evaluation results

\begin{tabular}{|l|c|c|c|c|c|}
\hline State & Analog & SD & Digital & SD & $p$ Value \\
\hline Anxiety & 5.4 & 4.06 & 3.6 & 2.16 & 0.103 \\
\hline Arousal & 3.6 & 3.37 & 3 & 2.63 & 0.642 \\
\hline Flow & 2.6 & 2.06 & 4 & 2.71 & 0.256 \\
\hline Control & 1.8 & 2.35 & 3.6 & 2.99 & 0.152 \\
\hline Relaxation & 7.8 & 3.49 & 4.8 & 2.97 & 0.053 \\
\hline
\end{tabular}

The on-task evaluation measured affective state on the Experience Fluctuation Model every 15 seconds throughout the activity. Anxiety and arousal were experienced more often by the analogue sketchers. Flow and control more often by the digital group. Relaxation was experienced more often by the analogue group. States of boredom, apathy and worry were not notable results for the study. Figure 2 provides an example of how these states varied on task and related to generative moments for a digital sketcher.

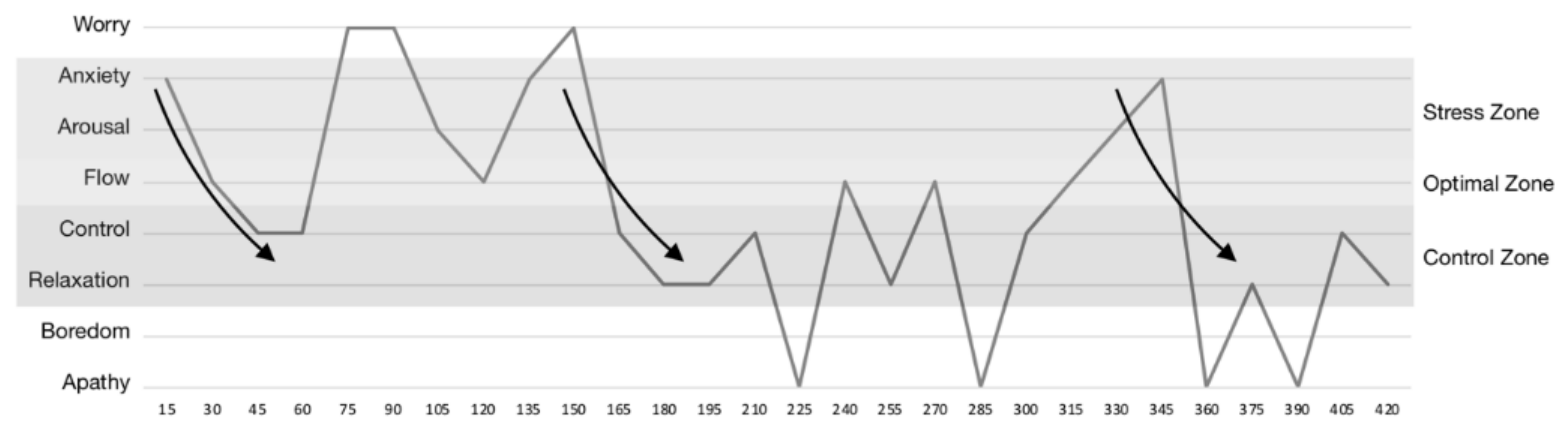

Figure 2. The example design flow fluctuations of digital sketcher D5

\subsection{End of Task Reflection Results}

Key results from the end of task reflection included perceived skill being rated lower in the analogue group ( $p=0.056)$. Absorption was rated higher in the digital group (mean $=4.6)$ compared to analogue $($ mean $=4)$ presenting a significant result $(p=0.037)$. Ratings for timelessness and effort to focus were not significant.

\subsection{Productivity Results}

Table 3. Comparison of on-task flow and productivity results

\begin{tabular}{|c|c|c|c|c|c|c|c|}
\hline & Flow & Fluency & Elaboration & & Flow & Fluency & Elaboration \\
\hline A1 & 2 & 6 & 7 & D1 & 6 & 4 & 4 \\
\hline A2 & 8 & 5 & 9 & D2 & 3 & 4 & 0 \\
\hline $\mathbf{A 3}$ & 0 & 5 & 8 & D3 & 5 & 2 & 2 \\
\hline A4 & 4 & 4 & 5 & D4 & 2 & 3 & 4 \\
\hline A5 & 4 & 2 & 3 & D5 & 5 & 2 & 5 \\
\hline A6 & 1 & 5 & 9 & D6 & 4 & 1 & 8 \\
\hline A7 & 0 & 3 & 5 & D7 & 1 & 5 & 5 \\
\hline A8 & 2 & 3 & 5 & D8 & 10 & 2 & 0 \\
\hline A9 & 5 & 4 & 9 & D9 & 1 & 2 & 6 \\
\hline A10 & 0 & 5 & 11 & D10 & 3 & 5 & 6 \\
\hline
\end{tabular}


The analysis of sketching fluency found that the analogue sketchers produced more ideas (mean $=4.2$ ) compared to the digital group $($ mean $=3)(p=0.057)$. However, elaboration was found to be significantly $(p=0.006)$ higher in the analogue group $($ mean $=7.5)$ compared to digital $($ mean $=4)$.

\section{Discussion}

Pre-task evaluation results are not significant. Mean values suggest digital and analogue groups viewed the task similarly, but the analogue group's slightly higher perceived task difficulty, lower sense of control, and lower skill belief may suggest they were less confident. Students who are digital sketchers have made recent and often relatively significant financial investments in sketching tools which may reflect an increased level of interest or confidence in using their tools.

The post task evaluation results found that the digital group found it easier focus, were less selfconscious, less distracted, had clearer goals, felt more in control and were more intrinsically motivated during the task. In addition, two separate measures showed that the digital group experienced a larger distortion of time, indicative of flow [12].

The aggregate on-task results for the digital group had a higher number of flow experiences and they had stronger flow ratings for nine of the twelve aspects evaluated post-task. A time perception test [12] cross-referenced with Mayer's Flow Scale [13] assessment showed that the digital group experienced a greater sense of timelessness. However, results for each groups' rating of direct feedback suggests that this flow pre-condition was not met in either group. This may have been due the fact that despite a stated aim to generate product ideas that 'stand out', participants literally did not receive any feedback on their progress towards this aim. With an unfamiliar brief, it may also have been difficult for participants to assess progress. Dorta et al. [10] established that designers who did not depend on second opinions experienced flow more easily. There was also no target for number of concepts, however fluency had been decided as a productivity measure for each tool/participant; any target could provide a selffulfilling prophecy and mask participants' performance.

The digital group found it easier to focus and were less distracted; could the digital interface and screen be more engaging and immersive than a piece of paper? Flow theory is now considered in software design [9] and one aspect mentioned by digital sketchers was that they found themselves switching tools or changing brush size during reported stress states; often when ideas had not emerged. Analogue sketchers were more likely to stare at the ceiling or re-read the brief. We might reflect on what counts as distraction in an ideation session. Arguably the designer's focus should be on exploring ideas not selecting tools. Fluency and elaboration results show that analogue sketchers were overall more prolific and detailed, which may suggest that current digital sketching software is not to be optimised for productivity in ideation activities. This conclusion reflects a similar perspective to Self et al. [4] who found that digital sketchers become tool-focused and not design-focused.

The on-task evaluation shows analogue sketchers reporting higher anxiety and arousal. Some level of stress may be beneficial over deeper engrossment in the task; entering flow seems to have caused digital sketchers to focus on developing ideas, rather than moving on. This could be related to a pleasurable sensation of flow, which people want to maintain or re-experience quickly [7]. The higher occurrence of the 'control' state in the digital group may support this.

In design flow theory a higher volume of ideas suggests that the designer has gone through more design flow cycles (see figure 2). We would expect a productive designer to experience more occurrences of the stress zone (anxiety and arousal), optimal zone (flow) and control zone (control and relaxation). Ontask evaluation suggested that the analogue group, experienced higher occurrence of anxiety, arousal and relaxation than the digital group. The design flow cycles for analogue sketchers are shorter, as they are generating more ideas within the same 7 minutes. This observation leads to contemplation that in order to generate more ideas, the designer should spend less time in flow.

Referring back to the data in Table 3, it is interesting that participant D8, who reported most flow states only produced two ideas, with no elaboration. However, an overview of the data shows that this does not apply to all instances. To verify these results more thoroughly, conducting a similar the experiment using the full Design 2 flow would be preferred, as the data is obtained with increased granularity [11]. The end of task reflection results identified that the perceived challenge of the activity remained constant for the digital sketchers before and after the task. For the analogue sketchers this dropped marginally suggesting the task was less difficult than expected. This could be linked to the higher volume of ideas. However, it was found that perceived skill dropped by 0.7 in the analogue group after the task was 
complete, whist the digital group only dropped by 0.1 . This indicates that the perceived confidence of the analogue group dropped more than for digital sketchers. As perceived skill is concerned with task performance; this result could be related to the number of sketches generated or the visual quality of the sketches produced. As the analogue sketchers produced a higher volume ideas on average, this could suggest that the visual quality of their sketches has some influence on the designers perceived skill.

The end of task reflection found that the digital group were more absorbed during the task. Similarly, the digital group experienced a greater sense of timelessness and used less effort to stay focused. This result reflects the findings of the previous evaluations which found that the digital group showed higher indicators of flow characteristics. Moreover, the result for timelessness, with was taken 10 minutes after the post-task evaluation, again shows the digital group experienced greater distortion of time. The results indicate that digital sketching tools may create better conditions for flow.

\section{CONCLUSIONS}

The aim of the study was to establish how the choice of sketching tool influences the designer's flow and identify the resulting impact this had on ideation productivity. In a study of twenty student designer's using either an analogue or digital sketching tool, it found that digital sketching tools appeared to create a more immersive sketching environment that led to longer flow experiences than analogue sketching tools. In addition, it proposed that increased flow negatively impacts task productivity when the goal of the task is to generate a high volume of ideas, though more work is required to fully establish this theory. To conclude, the contribution to knowledge is that digital sketching tools are not yet optimised for initial ideation activities and that the designer's cognitive experience is influenced by the sketching tool selected. For design educators, this means teaching traditional sketching remains valuable for initial ideation activities, as they form the foundation of later design development phases. Furthermore, it would suggest that educators should place more emphasis on developing digital skills which are becoming more valuable to later stages of concept development where long periods of concentration are required to deliver high quality renders or detailed sketches. Finally, this research shows there is large scope for exploring the influence of design tools further and indicates that this could be a valuable area of design research.

\section{REFERENCES}

[1] Jonson B. Design Ideation: The Conceptual Sketch in the Digital Age. Design Studies. November 2005, 26 (6), pp. 613-624.

[2] Coyne R., Hoon P. and Wiszniewski D. Design Devices: Digital Drawing and the Pursuit of Difference. Design Studies. Philosophy of Design. May 2002, 23 (3), pp. 263-286.

[3] Evans M. and Aldoy N. Digital Design Sketching Using the Tablet PC. The Design Journal. September 2016, 19 (5), pp. 763-787.

[4] Self J. et al. A Comparison of Digital and Conventional Sketching: Implications for Conceptual Design Ideation. Journal of Design Research. 2016, 14 (2).

[5] Csikszentmihalyi M. Finding Flow: The Psychology of Engagement with Everyday Life. New York: BasicBooks. 1997, p116-130.

[6] Heine C. Flow and achievement in mathematics. 1996, Unpublished doctoral dissertation, University of Chicago.

[7] Csikszentmihalyi M., Rathunde K. and Whalen S. Talented teenagers. 1993. Cambridge, England: Cambridge University Press.

[8] Nakamura J. and Csikszentmihalyi M. The concept of flow. In Snyder, C. R., \& Lopez, S. J. (Ed.). Oxford handbook of positive psychology. Oxford University Press, USA. 2009, pp. 89-105.

[9] Antonaci A. et al. Get Gamification of MOOC Right! International Journal of Serious Games. 2018, 5(3), pp. 61-78.

[10] Dorta T., Pérez E. and Lesage A. The ideation gap. Design Studies. 2008, 29(2), pp. 121-141.

[11] Safin S. et al. Design Flow 2.0, Assessing Experience during Ideation with Increased Granularity: A Proposed Method. Design Studies. November 2016, 47, pp. 23-46.

[12] Keller J. and Bless H. Flow and Regulatory Compatibility: An Experimental Approach to the Flow Model of Intrinsic Motivation. 2008.

[13 Mayers P. Flow in adolescence and its relation to the school experience. 1978. Unpublished doctoral dissertation, University of Chicago. 